\title{
Characterization of the carbon component in cometary Stardust samples by means of infrared and Raman spectroscopy
}

\author{
G. M. Muñoz Caro ${ }^{1}$, E. Dartois ${ }^{2}$, and K. Nakamura-Messenger ${ }^{3}$
}

\author{
${ }^{1}$ Centro de Astrobiología (CSIC-INTA), Carretera de Ajalvir, km 4, Torrejón de Ardoz, 28850 Madrid, Spain \\ e-mail: munozcg@inta.es \\ 2 Institut d'Astrophysique Spatiale, UMR 8617, Bât. 121, Université Paris XI, 91405 Orsay, France \\ ${ }^{3}$ Engineering Science Contact Group, NASA Johnson Space Center, Houston, TX 77058, USA
}

Received 19 October 2007 / Accepted 1 April 2008

ABSTRACT

\begin{abstract}
Aims. We attempt to ellucidate the structure and chemical composition of the carbon bulk detected in cometary Stardust particles. We determine if the carbon material observed spectroscopically is of true cometary origin and whether or not it was formed by direct UV-photoprocessing of icy grain mantles in the local dense cloud and/or the solar nebula.

Methods. We acquire infrared spectroscopy of ten Stardust cometary particles from track 35 and the aerogel inside and outside the particle track. Using infrared and Raman spectroscopy, the dominant carbon component in cometary Stardust particles was compared to IDPs and organics made from UV-photoprocessing of interstellar/circumstellar ice analogs in the laboratory. The Raman spectra of Stardust particles used in this comparison are adapted from the literature.

Results. As indicated in previous works, it is found that the collecting aerogel medium, processed during particle impact, poses serious problems for the infrared analysis of the Stardust cometary particles reported in this paper. We identify the structure of the carbon bulk of the organic material retrieved from the aerogel with a form of (hydrogenated) amorphous carbon. It is found that this material is not a direct product of ice photoprocessing.
\end{abstract}

Key words. comets: individual: 81P/Wild 2 - methods: analytical - techniques: spectroscopic

\section{Introduction}

The preliminary results from the examination of cometary Stardust particles are providing breakthroughs in our knowledge of the early Solar System and comets. The presence of high-temperature mineral grains ejected from the nucleus of comet $81 \mathrm{P} /$ Wild 2 and collected by Stardust, suggests that these minerals were formed in the inner regions of the solar nebula and later transported to the outer and colder regions (Brownlee et al. 2006). This implies that an important fraction of cometary mineral grains are not of interstellar origin. This was already suspected due to the predominance of crystalline silicates in comet nuclei detected by astronomical observations (Bregman et al. 1987; Hanner 1999).

In the Greenberg model for the formation of a comet nucleus, the organics present in comets are produced by UV-irradiation of icy-grain mantles in the interstellar medium (e.g. Greenberg 1998). Such ice photoprocessing was probably more significant in the solar nebula stages (Muñoz Caro \& Schutte 2003; Muñoz Caro et al. 2006). The organics produced by ice photoprocessing are of prebiotic interest (e.g. Muñoz Caro et al. 2002; Bernstein et al. 2002; Meierhenrich et al. 2004); if present in comets, they were probably delivered to the early Earth before life emerged (Oró 1961). It was proposed that the organic products of ice photoprocessing, which originate in the local dense cloud or the solar nebula, could be preserved in cometary nuclei that did not experience heating at temperatures above $300{ }^{\circ} \mathrm{C}$, and could be the precursors of the $\mathrm{D}$ and ${ }^{15} \mathrm{~N}$-enriched carbon component of IDPs and carbonaceous chondrites (Muñoz Caro et al. 2006, and ref. therein; Muñoz Caro \& Martínez-Frías 2007). Anomalies of D/H and ${ }^{15} \mathrm{~N} /{ }^{14} \mathrm{~N}$ prove that there are indigenous organics in some of the Stardust samples (McKeegan et al. 2006; Matrajt et al. 2007).
Organic matter enriched in ${ }^{15} \mathrm{~N}$, which was found in a Stardust sample, has a hollow globule shape similar to ones found in the Tagish Lake meteorite (Nakamura-Messenger et al. 2006), which might indicate formation by processing of ice mantles.

By comparison of infrared data of carbonaceous material present in diffuse interstellar grains with data of Stardust organics, Sandford et al. (2006) suggested that Stardust organics are not the direct result of stellar ejecta or diffuse interstellar medium processes but are produced instead by dense cloud and/or protosolar nebular processes. They continued to argue that the high $\mathrm{O}$ and $\mathrm{N}$ contents, lower aromatic contents, and elevated $-\mathrm{CH}_{2}-/-\mathrm{CH}_{3}$ ratios are all qualitatively consistent with what is expected from radiation processing of astrophysical ices and the polymerization of simple species such as $\mathrm{HCO}, \mathrm{H}_{2} \mathrm{CO}$, and HCN (Sandford et al. 2006).

It was shown by Spencer \& Zare (2007) that laser impacts on aerogel, which has not been exposed to cometary particles, can generate complex aromatic molecules from low-mass carbon impurities present in the aerogel. This study demonstrated the difficulty in distinguishing between cometary and noncometary organic compounds, which occupy particle-impact tracks in aerogel. Sandford \& Brownlee (2007) reiterated that the effects of the contamination and alteration of the samples, must be carefully considered.

Using infrared spectroscopy, we characterize the carbon bulk of ten cometary Stardust particles, extracted from track 35. In particular, we address aerogel contamination in our samples, which corresponds to absorptions in the infrared, previously discussed (Sandford et al. 2006; Keller et al. 2006). We attempt to provide a more accurate interpretation of the Raman spectra of Stardust grains, which was reported by Sandford et al. (2006). It 


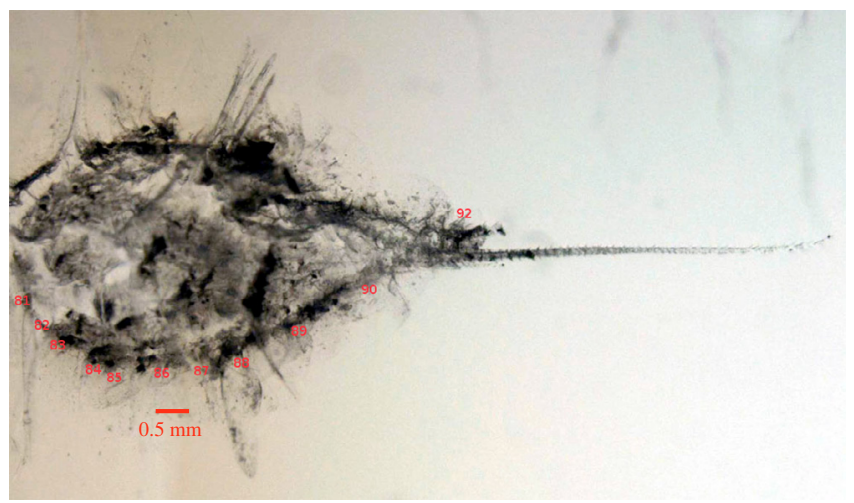

Fig. 1. Track 35 showing the position of the particles studied in this work (red numbers). Particles 81 to 90 were extracted from the lower edge of the bulb and particle 92 from the upper edge.

is shown that the infrared and Raman spectra that infer the presence of carbon in Stardust samples, and of carbon in most IDPs, are rather similar. Therefore, for the interpretation of the spectra of Stardust samples we can therefore use the results from our previous study of the characterization of the carbon bulk in IDPs (Muñoz Caro et al. 2006).

The infrared spectra of Stardust samples are compared to those of organic residues made from ice photoprocessing, prior and after annealing at different temperatures. In this way, we were able to study the possibility that the carbon bulk in Stardust grains, originated from UV-irradiation of icy grain mantles in dense clouds and/or the solar nebula, as suggested by Sandford et al. (2006).

\section{Experimental}

The ten Stardust cometary grains used for this project were provided by the NASA-JSC curation facility. Their sizes are in the range $10-20 \mu \mathrm{m}$. All grains belonged to particle track 35: they are named C2054, 0, 35, X, 0, where $X=81-87,89$, 90, or 92; henceforth, they are referred to by their $X$ number. A picture showing the particle positions in track 35, is given in Fig. 1. Track 35 is one of the largest tracks collected by Stardust, having a length of $11.7 \mathrm{~mm}$ and a bulbous shape. There are only 10 other tracks of this size, and Track 35 is the only one of these large tracks extracted so far. Since it has well-developed bulb shape, the original impacting particle was probably made up of fine grained, volatile-rich material. Upon impact into the aerogel, the material was rapidly disaggregated and volatile-rich material exploded, creating the large bulb at the top of the track. The largest mineral grain traveled the farthest, reaching $11.7 \mathrm{~mm}$. The 10 grains allocated to us from track 35 are from the bulbous region (Fig. 1), and thus most likely contain a larger amount of organic matter, in comparison to carrot-shaped cometary tracks (Sandford et al. 2006; Hörz et al. 2006; Domínguez et al. 2007). An additional contributor to track bulb production could be particle fragmentation during capture. For instance, laboratory work, shooting projectiles into aerogel, showed that bulbous cavities can also be generated because of the low cohesive strength of a fine-grained matrix (e.g. Hörz et al. 1998, 2006). More laboratory analyses of samples are required to establish whether evaporation of volatile phase in comet particles, was responsible for the bulbous part formation; if it was, then places kinetic constraints on the survival of carbon and/or hydrocarbon materials in the tracks.

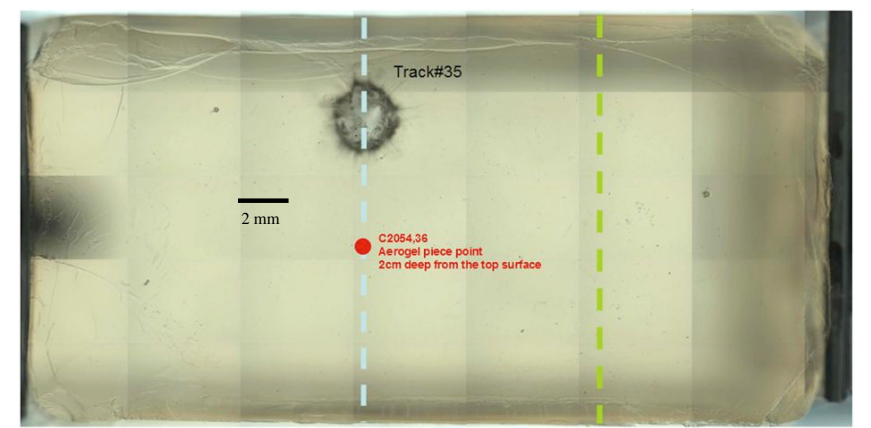

Fig. 2. Slice of Stardust aerogel tile C2054 showing track 35 and the position where the aerogel piece away from the track, C2054, 36, was extracted. This position, indicated by the red dot, is more than $3 \mathrm{~mm}$ away from the track and at $20 \mathrm{~mm}$ depth.

Sample preparation, which involves the extraction of grains from the aerogel and the crushing of them onto a $\mathrm{KBr}$ window, was performed by one of us (K.N.-M.). The grains were extracted, embedded on a piece of aerogel of variable size, due to the difficulty of isolating grains from the aerogel. While crushing the grains onto the $\mathrm{KBr}$ window, the aerogel surrounding the grain is therefore also crushed and is sometimes observed next to the grain. For comparison with the samples inside the track, we extracted an aerogel piece, C2054, 36, from a position more than $3 \mathrm{~mm}$ away from the track, on the top surface, shown in Fig. 2, and $20 \mathrm{~mm}$ deep from the top surface. As mentioned above, the length of track 35 is $11.7 \mathrm{~mm}$, and therefore the aerogel blank was $\sim 8 \mathrm{~mm}$ deeper than the tip of the bulb.

The experimental protocol used for infrared (FT-IR) spectroscopy and the basic concepts required for the interpretation of both infrared and Raman spectral data are summarized below.

\subsection{FT-IR analysis}

Fourier-transform infrared (FT-IR) spectroscopy was completed using the Nicolet Magna-IR 560 ESP spectrometer, coupled to a Nicolet Nicplan infrared microscope, at the University of Paris-Sud, Orsay, France. The spectrometer is equipped with a $\mathrm{KBr}$ beamsplitter and a nitrogen-cooled MercuryCadmium-Tellure (MCT) detector, which works in the 4000 to $650 \mathrm{~cm}^{-1}(2.5$ to $15 \mu \mathrm{m})$ spectral range. The microscope uses a Schwarzchild-Cassegrain objective (32x) and a condenser (10x), in the transmission mode. We used the standard internal light source of the instrument, a conventional black-body-like source (Globar). The collected spectra were the average of 50-100 acquisitions of 64 scans, each of a resolution $4 \mathrm{~cm}^{-1}$.

The infrared spectra of Stardust samples were usually corrected for light-scattering effects, using a spline baseline, through regions where there were no absorption features. Integration of the absorbance area of the features was performed using an in-house IDL code.

The column densities of the features associated with the C-H stretching of aliphatics, around $3.4 \mu \mathrm{m},(N[3.4]$, in aliphatic $\mathrm{C}$ atoms $\left.\mathrm{cm}^{-2}\right)$, or the $\mathrm{Si}-\mathrm{O}$ stretching in silicates/aerogel, around $10 \mu \mathrm{m}\left(N[10]\right.$, in $\mathrm{Si}$ atoms $\left.\mathrm{cm}^{-2}\right)$, can be determined using the formula

$N=\int_{\text {band }} \frac{\tau_{\nu} \mathrm{d} v}{A}$

where $N$ is the column density in $\mathrm{cm}^{-2}, \tau$ is the optical depth of the band, $\mathrm{d} v$ is the wavenumber differential in $\mathrm{cm}^{-1}$, and $A$ is the band strength in $\mathrm{cm} /$ molecule. 
We calculated the column density of aliphatic $\mathrm{C}$ atoms to be $N[3.4]=N\left(\mathrm{CH}_{2}\right)+N\left(\mathrm{CH}_{3}\right)$, where the absorbance areas of the bands at 2958 and $2920 \mathrm{~cm}^{-1}$ (asymmetric $\mathrm{CH}_{3}$ and $\mathrm{CH}_{2}$ stretching modes) were calculated using Gaussian fits. This provided the ratio area $\left(\mathrm{CH}_{2}\right) / \operatorname{area}\left(\mathrm{CH}_{3}\right)$. For the calculation of $N\left(\mathrm{CH}_{2}\right) / N\left(\mathrm{CH}_{3}\right)$, we adopted the band strengths for hexane ice (Dartois et al. 2004), $A\left(\mathrm{CH}_{3}\right)=1.25 \times 10^{-17} \mathrm{~cm}(\mathrm{C} \text { atom })^{-1}$ and $A\left(\mathrm{CH}_{2}\right)=8.4 \times 10^{-18} \mathrm{~cm}(\mathrm{C} \text { atom })^{-1}$. For particles 82 and 90 , the poor signal-to-noise ratio of the $3.4 \mu \mathrm{m}$ feature did not enable determination of $N\left(\mathrm{CH}_{2}\right)$ and $N\left(\mathrm{CH}_{3}\right)$, and thus $N$ [3.4] was obtained by completing an integration between $3000-2800 \mathrm{~cm}^{-1}$, using the band strength for hexane ice $A[3.4]=1.2 \times 10^{-17} \mathrm{~cm}$ $(C \text { atom })^{-1}$ (Dartois et al. 2004).

For the evaluation of the column density of the Si-O stretching band, in silicates/aerogel around $\sim 1000 \mathrm{~cm}^{-1}$ or $\sim 10 \mu \mathrm{m}$ $(N[10]), A[10]=2.0 \times 10^{-16} \mathrm{~cm}(\mathrm{Si} \text { atom })^{-1}$ was used.

\subsection{Raman analysis}

Raman spectroscopy provides information on the chemical composition and structure of carbonaceous samples, which makes it an invaluable tool for probing the evolution of the material due to the effect of irradiation and/or thermal annealing (Tuinstra \& Koening 1970). Progress in the theoretical description of Raman spectroscopy of carbonaceous materials, and in particular the work of Ferrari \& Robertson (2000), allows a more precise determination of the structure of carbonaceous solids. Based on Ferrari \& Robertson (2000), we provided a characterization of the carbon bulk in IDPs (Muñoz Caro et al. 2006). A similar characterization can be attained for Stardust samples. This section outlines the basic method (adapted from Ferrari \& Robertson 2000) required to complete the Raman characterization of the carbon bulk in IDPs and Stardust samples.

Microcrystalline graphite has a single line at $1582 \mathrm{~cm}^{-1}$ (graphitic or $G$ line, assigned to zone-center phonons of $E_{2 g}$ symmetry). Disordered graphite shows two lines around $1350 \mathrm{~cm}^{-1}$ (disorder or D line, assigned to $K$-point phonons of $A_{1 g}$ symmetry) and $1580-1600 \mathrm{~cm}^{-1}$ (G line). The full width at half maximum (FWHM) of the D band is related to the degree of graphitisation of the carbonaceous material: as the degree of graphitisation increases, the FWHM of the D band decreases. The position of the $\mathrm{G}$ band is related to the structural type of the carbon (nanocrystalline, polycrystalline, or amorphous) and, similar to the D band, its FWHM increases as the degree of order decreases. The intensity ratio of the first order $\mathrm{D}$ and $\mathrm{G}$ lines, $I(\mathrm{D}) / \mathrm{I}(\mathrm{G})$, is given by

$$
\frac{I(\mathrm{D})}{I(\mathrm{G})}=\frac{C(\lambda)}{L_{\mathrm{a}}}
$$

where $L_{\mathrm{a}}$ is the aromatic cluster diameter or in-plane correlation length and $C(515.5 \mathrm{~nm}) \sim 44 \AA$. This relation, however, is not valid for highly-disordered carbon materials (Tuinstra \& Koenig 1970).

The effects of the amorphisation of graphite on the evolution of the Raman spectrum, following the sequence graphite $\rightarrow$ nanocrystalline graphite $\rightarrow$ amorphous carbon $\rightarrow$ tetrahedral amorphous carbon, were discussed by Ferrari \& Robertson (2000). The particular effects on Raman spectroscopy that these authors observed, as nanocrystalline graphite evolves towards amorphous carbon $(a-\mathrm{C})$, were

(a) The $G$ peak decreases from 1600 to $\sim 1510 \mathrm{~cm}^{-1}$; (b) Eq. (2) is no longer valid. Instead

$$
\frac{I(\mathrm{D})}{I(\mathrm{G})}=C^{\prime}(\lambda) \cdot L_{\mathrm{a}}^{2}
$$

where $C^{\prime}(514 \mathrm{~nm}) \approx 0.0055 \AA^{-2}$.

(c) $I(\mathrm{D}) / \mathrm{I}(\mathrm{G})$ goes from 2 to values close to zero.

(d) Increasing dispersion of the $G$ peak occurs. The Raman spectra of hydrogenated amorphous carbon $(a-\mathrm{C}: \mathrm{H})$ present first-order peaks similar to amorphous carbon $(a-\mathrm{C})$, unless they are masked by photoluminescence. For $a-\mathrm{C}$, the $\mathrm{sp}^{3}$ content varies from almost 0 to close to $10 \%$ (see Fig. 7 of Ferrari \& Robertson 2000), while for $a-\mathrm{C}: \mathrm{H}$ the $\mathrm{sp}^{3}$ content could be, roughly, up to about $30 \%$.

Ferrari \& Robertson (2000) conclude that the G mode is related to the in-plane bond-stretching motion of pairs of $\mathrm{C} \mathrm{sp}{ }^{2}$ atoms. This mode does not require the presence of sixfold rings. The label G (for "graphitic") is therefore unfortunate, because this mode occurs in any $\mathrm{sp}^{2}$ paired carbon atoms. It occurs at all $\mathrm{sp}^{2}$ sites, not only those in rings, and can therefore occur in olefinic chains. The D peak is a breathing mode of $A_{1 g}$ symmetry which involves phonons close to the $K$ zone boundary, that becomes active in the presence of disorder; its position depends on the photon excitation energy. Ferrari \& Robertson (2000) showed that the intensity of the D peak is strictly connected to the presence of sixfold aromatic rings.

\section{Experimental results}

We present and discuss the results obtained from our analysis of infrared spectroscopy of Stardust grains. In particular, attention is devoted to the spectroscopic interferences caused by aerogel, in our attempt to constrain the level of contamination experienced by the samples. By means of infrared and Raman spectroscopy, Stardust grains are compared to both IDPs and organic residues made from UV-photoprocessing of interstellar/circumstellar ice analogs. The Raman spectra of Stardust grains used for such a comparison were adapted from Sandford et al. (2006).

\subsection{Characterization of Stardust grains by infrared spectroscopy}

Unfortunately, aerogel, the collecting medium, was found to absorb in the main infrared regions of interest for the case of organic and silicate grains: the $3.4 \mu \mathrm{m}$ region, $\mathrm{C}-\mathrm{H}$ stretching modes of aliphatics, and around $10 \mu \mathrm{m}, \mathrm{Si}-\mathrm{O}$ stretching mode (e.g. Sandford et al. 2006; Keller et al. 2006). Sandford et al. (2006) found that the organic components that absorb in the mid-infrared extend well beyond the visible edge of the track, which suggests that the incoming particles contained organics that volatilized during impact and diffused into the surrounding aerogel. They provided evidence that the observed absorption bands due to organics are unlikely to be created by impactaltered $\mathrm{C}$ from the aerogel.

Figure 3 shows particle 84 , which has been crushed onto a $\mathrm{KBr}$ window, with a piece of aerogel alongside, which was extracted at the same time. Spectra of both the particle and aerogel are shown in Fig. 4. The KBr infrared window, in which particle 84 is mounted, has no contamination features in the observed spectral region (Fig. 3); this excludes the possibility of any significant contamination of the $\mathrm{KBr}$ window. The spectrum of an aerogel piece is also shown in Fig. 4: this piece was extracted 


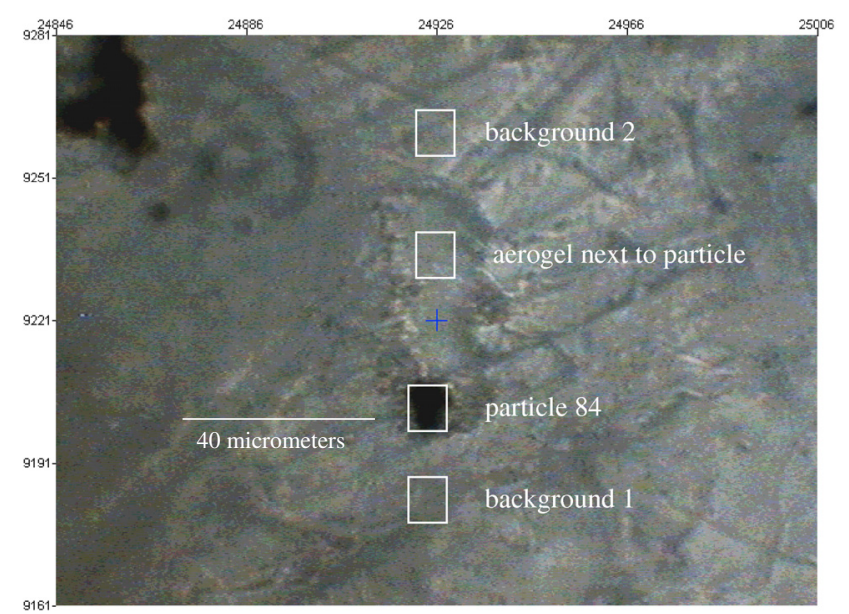

Fig. 3. Picture shows particle 84 as a dark spot positioned south of a $50 \mu \mathrm{m}$ long aerogel piece that was extracted with the particle. The squares indicate the apertures used for FTIR analysis; see spectra in Fig. 4.

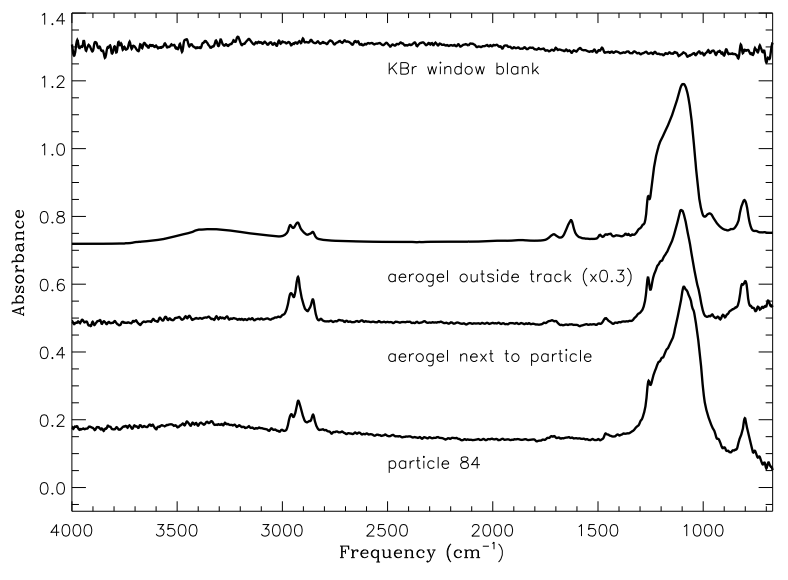

Fig. 4. From bottom to top traces: infrared spectrum of particle 84 , see Fig. 3 for sample and background apertures, resp. "particle 84" and "background 1". The spectrum of "aerogel next to particle" was made using "background 2". The spectrum of a piece of aerogel outside the particle track is also shown for comparison. The $\mathrm{KBr}$ window blank is made using "background 1" as sample and "background 2" as background.

from a region inside cell C2054, in which track 35 (the source of all the grains analyzed in this work) is located; the region was, however, well outside track 35 as described in Sect. 2. The $3.4 \mu \mathrm{m}$ feature of this aerogel has bands due to $-\mathrm{CH}_{2}-$ and $-\mathrm{CH}_{3}$ groups, with a $N\left(\mathrm{CH}_{2}\right) / N\left(\mathrm{CH}_{3}\right)$ ratio of 3.2. The spectrum of the aerogel piece next to particle 84 (see Fig. 3) and the spectrum of particle 84 itself, have also a $N\left(\mathrm{CH}_{2}\right) / N\left(\mathrm{CH}_{3}\right)$ ratio of 3.2. The $10 \mu \mathrm{m}$ features of particle 84 and the aerogel next to the particle are also practically identical and similar to that of the aerogel piece outside the track. This indicates that the infrared spectrum of particle 84 contains an important contribution from components present in the aerogel where the particle is embedded, which is difficult to remove. Similar results were obtained for other particles for which the surrounding aerogel was analyzed $(86,87$, and 90). The fact that the aerogel piece removed from a region away from the track showed a similar spectrum to the track particles was surprising, as one might expect that the $3.4 \mu \mathrm{m}$ feature due to hydrocarbons of possible cometary origin inside the track gradually changes its profile, and turns into the

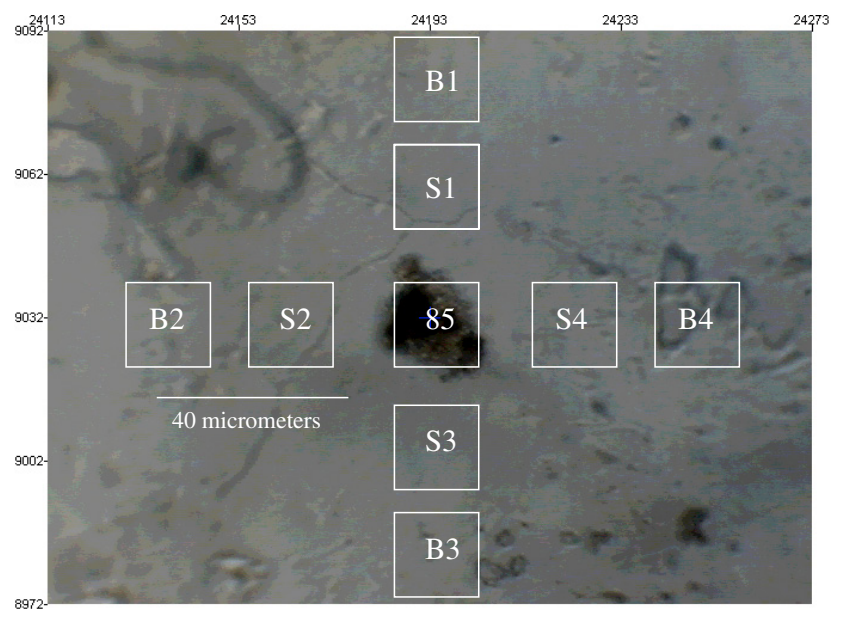

Fig. 5. Picture shows particle 85 . The squares indicate the apertures used for FTIR analysis, see spectra in Fig. 6.

$3.4 \mu \mathrm{m}$ feature characteristic of aerogel, as observed by Sandford et al. (2006). As mentioned above, Sandford et al. (2006) observed absorption due to organics well beyond the visible edge of the track. Apparently, the distance at which we extracted the aerogel piece outside the track, was not sufficiently distant for significant differences in the spectrum to be observed. The probability that we detected the halo of organics that volatilized during impact and diffused into the aerogel surrounding track 35 is rather low, because the distance at which we extracted the aerogel piece outside the track was at least $3000 \mu \mathrm{m}$ away from the track walls (see Sect. 2), compared to $125 \mu \mathrm{m}$ in Fig. 2 of Sandford et al. (2006) for track 59.

Particle 85 was the only particle for which the $3.4 \mu \mathrm{m}$ feature was detected exclusively at the position of the particle, and not in the vicinity of the particle. Figure 5 shows the sample and background aperture positions for the spectra shown in Fig. 6. Particle 85 shows the characteristic 3.4 and $10 \mu \mathrm{m}$ features. The spectra of the regions surrounding particle 85 have a $\sim 20$ times lower absorbance than the spectrum corresponding to the particle position; they do not show evidence of a $3.4 \mu \mathrm{m}$ feature, only the $10 \mu \mathrm{m}$ feature being present. Given the low absorbance value of the $10 \mu \mathrm{m}$ feature in the surrounding aerogel, the intrinsically weaker $3.4 \mu \mathrm{m}$ feature is probably, however, below the detection limit.

Figure 7 shows the spectra of all particles studied in this work; the figure caption indicates the particle assignment of each spectra. All spectra are rather similar and resemble the spectrum of the above mentioned aerogel piece that was on the same cell as track 35, but away from any tracks (top spectrum). As mentioned above, this indicates that the observed absorption in the mid-infrared is mainly due to matter present in the aerogel, which can be either captured carbonaceous and mineral matter of cometary origin, or material indigenous to the aerogel. The spectrum of particle 85 (fifth from the bottom in Fig. 7), the only particle for which no $3.4 \mu \mathrm{m}$ feature was observed in the surrounding aerogel, is similar to that of the other grains; it could therefore have been produced by the particle, although it is impossible to measure how much aerogel there would have been on top and beneath the particle. The profile of the $10 \mu \mathrm{m}$ feature varies between particles. Such spectral variations may be indicative of the various combinations of cometary silicate grains and aerogel present, although scattering effects and/or different degrees of processing of the aerogel experienced during particle impact could have a significant effect. Scattering effects, when 


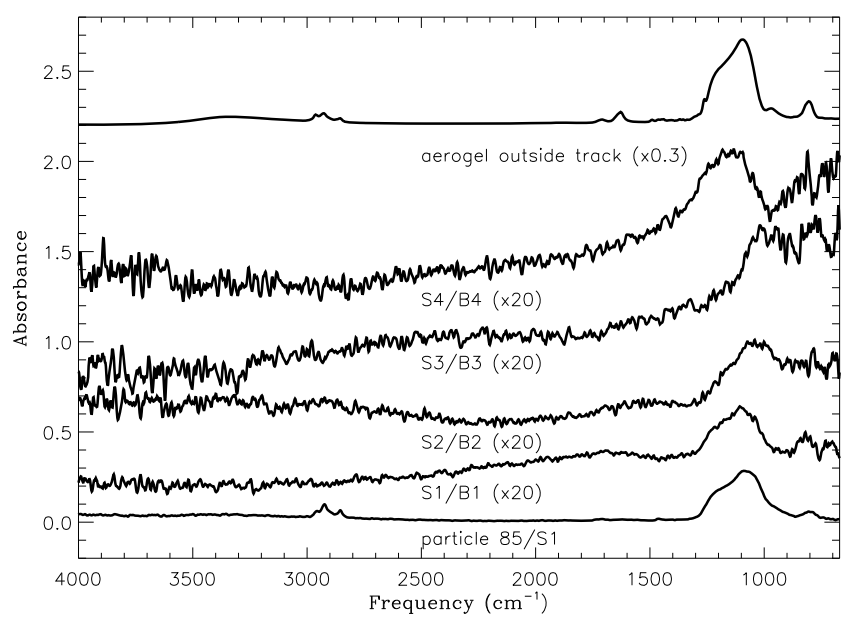

Fig. 6. From bottom to top traces: infrared spectrum of particle 85 , see Fig. 3 for sample and background apertures, resp. " 85 " and "S1". The other spectra were measured using positions $\mathrm{S} 1$ to $\mathrm{S} 4$ as samples and B1 to B4 as their backgrounds. The spectrum of a piece of aerogel outside the particle track is also shown for comparison.

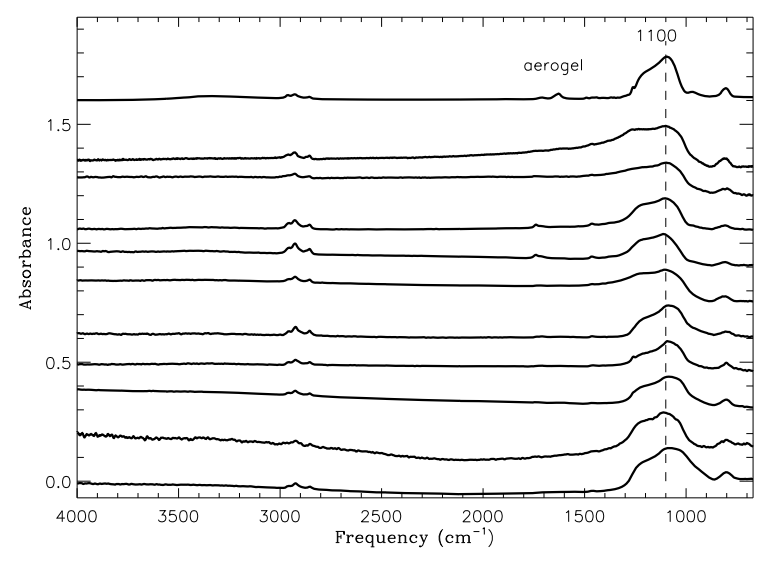

Fig. 7. From bottom to top traces: infrared spectra of Stardust particles 81-87, 89, 90, 92 extracted from track 35, see Fig. 1. Top spectrum corresponds to an aerogel piece that was outside the track. All spectra were normalized in intensity with respect to the bottom spectrum (particle 81$)$.

the grain size is close to the wavelength, are expected to affect the $10 \mu \mathrm{m}$ feature significantly (Raynal et al. 2000). Highly focused beam infrared spectroscopy, such as infrared synchrotron beamline, should help in minimizing potential scattering effects. A zoom of the $3.4 \mu \mathrm{m}$ feature of both the particles and the aerogel piece away from the particle track, is shown in Fig. 8, after a local background correction. Most spectra have a good signalto-noise ratio, apart from particles 82 and 90. For comparison, the $3.4 \mu \mathrm{m}$ feature of particle C2054, 0, 35, 16, 0 (particle 16 of track 35, adapted from Sandford et al. 2006) is shown (thin trace bottom spectrum); this particle was extracted from a region close to particle 89 , and its $3.4 \mu \mathrm{m}$ feature is similar to that of the particles analyzed in this work, which are shown in Fig. 8.

The experimental parameters of the spectra shown in Figs. 7 and 8 , are provided in Table 1 . These are the particle number, the column density of the $3.4 \mu \mathrm{m}$ feature in aliphatic $\mathrm{C}$ atoms $\mathrm{cm}^{-2}$, $N[3.4]$, the ratio between column densities of the $\mathrm{CH}_{2}$ and $\mathrm{CH}_{3}$ groups, $\frac{N\left(\mathrm{CH}_{2}\right)}{N\left(\mathrm{CH}_{3}\right)}$, and the ratio between column densities of

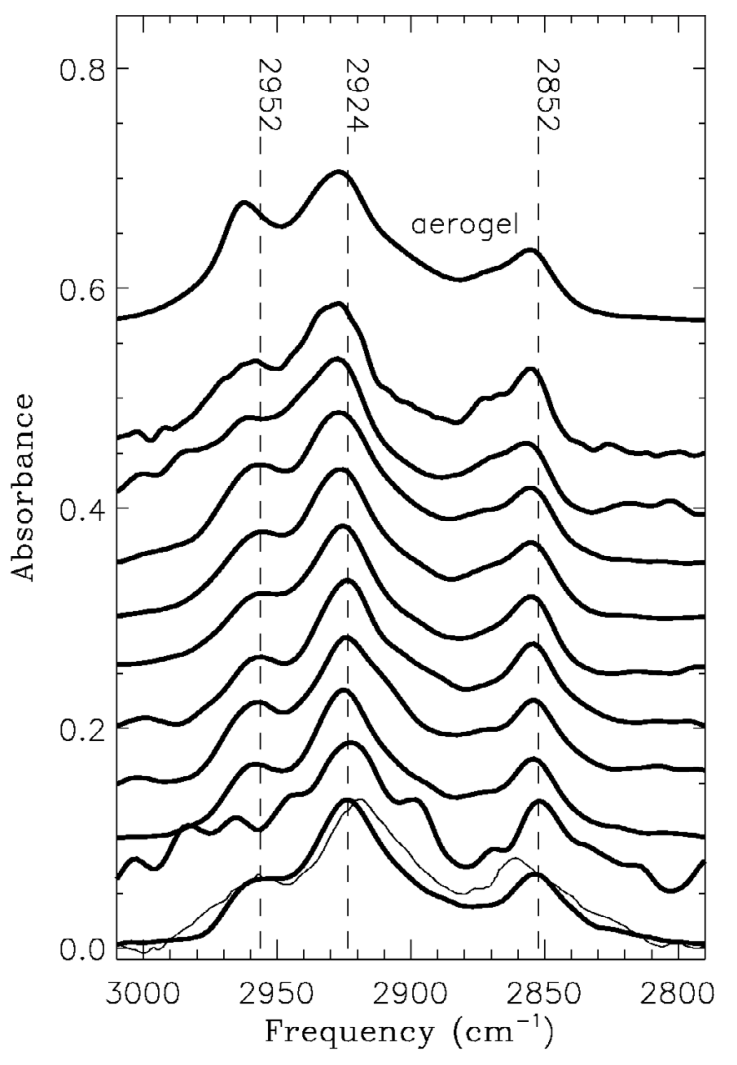

Fig. 8. From bottom to top traces: $3.4 \mu \mathrm{m}$ infrared feature of Stardust particles 81-87, 89, 90, 92 extracted from track 35, see Fig. 1. Top spectrum corresponds to $3.4 \mu \mathrm{m}$ infrared feature of an aerogel piece that was outside the particle track. Spectra were normalized in intensity with respect to the spectrum of particle 87 ( 7 th trace from bottom), the particle with the highest $N[3.4] / N[10]$ (aliphatic $\mathrm{C}$ to $\mathrm{Si}$ ratio). For comparison, the $3.4 \mu \mathrm{m}$ feature of Stardust particle C2054, 0, 35, 16, 0 is shown (thin trace, adapted from Sandford et al. 2006).

Table 1. Experimental parameters corresponding to particles used in this work. See text in Sect. 2.1 for explanation.

\begin{tabular}{cccc}
\hline \hline Particle & $\begin{array}{c}N[3.4] \\
\text { aliph. C cm }\end{array}$ & $\frac{N\left(\mathrm{CH}_{2}\right)}{N\left(\mathrm{CH}_{3}\right)}$ & $\begin{array}{c}\frac{N[3.4]}{N[10]} \\
\text { (aliph. C/Si) }\end{array}$ \\
\hline 81 & $1.4 \times 10^{17}$ & 4.2 & 0.31 \\
82 & $9.1 \times 10^{16}$ & - & 0.36 \\
83 & $1.4 \times 10^{17}$ & 3.4 & 0.33 \\
84 & $5.4 \times 10^{17}$ & 3.2 & 0.56 \\
85 & $4.1 \times 10^{17}$ & 3.7 & 0.67 \\
86 & $3.8 \times 10^{17}$ & 3.1 & 0.44 \\
87 & $7.0 \times 10^{17}$ & 2.9 & 0.70 \\
89 & $6.4 \times 10^{17}$ & 2.5 & 0.64 \\
90 & $7.9 \times 10^{16}$ & - & 0.23 \\
92 & $1.7 \times 10^{17}$ & 2.6 & 0.59 \\
Aerogel & $2.1 \times 10^{18}$ & 3.2 & 0.75 \\
\hline
\end{tabular}

the 3.4 and $10 \mu \mathrm{m}$ features, $\frac{N[3.4]}{N[10]}$, in aliphatic $\mathrm{C}$ atoms/Si atoms. The value of $\frac{N\left(\mathrm{CH}_{2}\right)}{N\left(\mathrm{CH}_{3}\right)}$ appears to decrease along the track, from 4.2 to 2.6, probably due to a gradient in temperature processing. Several Stardust grain tracks should be studied for evidence of similar trends. The value of $\frac{N\left(\mathrm{CH}_{2}\right)}{N\left(\mathrm{CH}_{3}\right)}$ for the aerogel sample outside the track, 3.2, is exactly the average value of the 8 particles that allowed the determination of the $\frac{N\left(\mathrm{CH}_{2}\right)}{N\left(\mathrm{CH}_{3}\right)}$ ratio. There is no clear trend in the values of the $\frac{N[3.4]}{N[10]}$ ratio for particles along the track, 
but they all fall below the value for the aerogel sample outside the track; thus, if we assume that aliphatic carbon, the carrier of the infrared $3.4 \mu \mathrm{m}$ feature, is of cometary origin, then one would expect to find more aliphatic $\mathrm{C}$ relative to $\mathrm{Si}$, inside the track than away from the track, and not otherwise as we actually observe.

It is possible, given the high velocities of the impacting cometary particles, that some of these particles will be altered and some of the carbon that was originally inside the aerogel, may be converted into different forms (Sandford \& Brownlee 2007, and ref. therein). Thus, aerogel carbon, processed during impact, is expected to coexist with the cometary material, and the infrared spectrum of impact-processed aerogel is, to the best of our knowledge, unknown. The $3.4 \mu \mathrm{m}$ features in our samples are similar to those reported by Sandford et al. (2006) and Keller et al. (2006), and are clearly distinct from that of the unprocessed aerogel onboard Stardust. In view of our results, it appears difficult to quantify the contribution of both aerogel and cometary material to the infrared absorption bands, which are within the processed aerogel region where the grains are collected.

A simple calculation can roughly estimate if carbon contamination, detected in the infrared, is expected to be a problem. The volume $V$ of the bulb produced in the aerogel by the particle impact (Fig. 1), can be approximated as an ellipsoid of revolution, and thus

$V=\frac{4}{3} \pi r^{2} h$

where $r$ is the semimajor axis and $\mathrm{h}$ is the semiminor axis, which have values of 0.345 and $0.135 \mathrm{~cm}$ respectively, giving $V=0.067 \mathrm{~cm}^{-3}$. For an aerogel density of $0.01 \mathrm{~g} \mathrm{~cm}^{-3}$, this volume corresponds to a mass of $6.7 \times 10^{-4} \mathrm{~g}$. Assuming that the carbon contamination in the aerogel is $0.5 \%$ by mass (Tsou et al. 2003), the mass of aerogel carbon in the bulb is $3.4 \times 10^{-6} \mathrm{~g}$. This mass is equivalent to a spherical particle made of pure carbon with density $2.2 \mathrm{~g} \mathrm{~cm}^{-3}$ of $144 \mu \mathrm{m}$ diameter, which is a very large particle size! In addition, the $0.5 \%$ by mass $C$ in the aerogel, measured by Tsou et al. (2003), is probably a lower limit since Sandford \& Brownlee (2007) reported, literally, a few weight percent $\mathrm{C}$ in the aerogel. The cometary grain that formed track 35 should exceed this particle size to overcome the problem of aerogel $\mathrm{C}$ contamination influencing the infrared spectra.

The aliphatic carbon in our samples, is in the range $\frac{N[3.4]}{N[10]}=$ 0.31-0.70 aliph. $\mathrm{C} \mathrm{cm}^{-2}$, as shown in Table 1; considering as an approximation that aliphatic $\mathrm{C}$ is in the form of $\mathrm{CH}_{2}(14 \mathrm{amu})$ groups and $\mathrm{Si}$ as $\mathrm{SiO}_{2}(60 \mathrm{amu})$, we obtain a carbon concentration of $0.31 \frac{14}{60}$ to $0.70 \frac{14}{60} \sim 7.2$ to $16.3 \%$ by mass in our samples, i.e. well above the $0.5 \%$ by mass carbon contamination measured by Tsou et al. (2003), and at least equivalent or higher than the "few weight percent $\mathrm{C}$ contamination" reported by Sandford \& Brownlee (2007). That carbon excess in our samples, compared to the expected carbon contamination, could in principle correspond to cometary carbon. On the other hand, Sandford et al. (2006) mention that the infrared features due to carbon matter, when present, are particularly intense along the track walls. Thus, another possibility is that carbon, in general, both cometary carbon and aerogel carbon contamination, concentrated on the track walls where our particles were located, as can be seen in Fig. 1. The conclusion is that the carbonaceous matter observed in our samples by infrared spectroscopy, is a mixture that contains cometary carbon and carbon from the aerogel, being difficult to determine quantitatively the contribution from both sources.

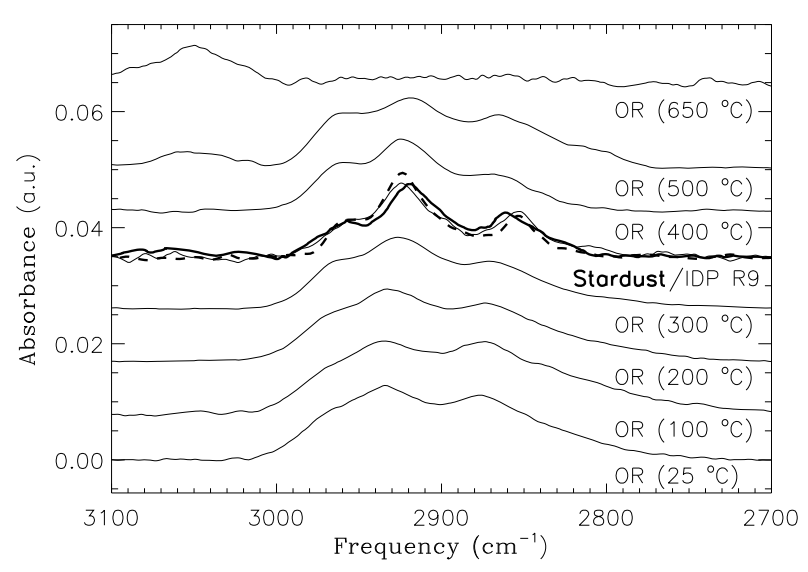

Fig. 9. 3.4 $\mu \mathrm{m}$ infrared feature due to $\mathrm{CH}$ stretching modes of organic residues (OR) made from ice photoprocessing at $12 \mathrm{~K}$ followed by annealing at different temperatures, and that of Stardust particles C2054, $0,35,16,0$ (thick trace, adapted from Keller et al. 2006) and 81 (thick dashed trace) overplotted on the same feature of an IDP (labelled R9, adapted from Muñoz Caro et al. 2006).

\subsection{Infrared comparison of cometary Stardust samples to organic residues made from UV-photoprocessing of interstellar ice analogs}

In the previous section, we discussed the issue of potential $\mathrm{C}$ contamination in our aerogel samples. Here we focus on the infrared comparison between cometary Stardust samples and organic residues made from UV-photoprocessing of interstellar ice analogs. Figure 9 shows the aliphatic $\mathrm{CH}$ stretch bands (3.4 $\mu \mathrm{m}$ feature) of Stardust particle C2054, 0, 35, 16, 0 (thick trace, adapted from Keller et al. 2006), and particle 81 (thick dashed trace) shown with the same feature of an IDP (L2036R9, abbreviated R9, adapted from Muñoz Caro et al. 2006). Using the method described in Sect. 2.1, the value estimation of area $\left(\mathrm{CH}_{2}\right) /$ area $\left(\mathrm{CH}_{3}\right)$ for particle $\mathrm{C} 2054,0,35,16,0$ is 2.8 , in agreement with the $\sim 2.5$ value reported for $81 \mathrm{P} /$ Wild 2 particles (Keller et al. 2006), or $N\left(\mathrm{CH}_{2}\right) / N\left(\mathrm{CH}_{3}\right)=4.2$, similar to the value of particle 81 (Table 1 ). As indicated by Keller et al. (2006), this value does not differ from the average ratio in IDPs. Using the same procedure for IDPs, it was found that $N\left(\mathrm{CH}_{2}\right) / N\left(\mathrm{CH}_{3}\right)=3.7 \pm 0.9$ (Matrajt et al. 2005), and the profile of the $3.4 \mu \mathrm{m}$ feature in IDPs with high $N\left(\mathrm{CH}_{2}\right) / N\left(\mathrm{CH}_{3}\right)$ ratios, such as $\mathrm{R} 9$, is similar to that of Stardust particles C2054, 0, 35, 16,0 (see Fig. 9) and 81. Other IDPs show lower $N\left(\mathrm{CH}_{2}\right) / N\left(\mathrm{CH}_{3}\right)$ ratios, which are similar to the other Stardust particles analyzed here (Table 1). The carbon bulk of most IDPs shows an infrared spectrum that corresponds to $a-\mathrm{C}: \mathrm{H}$ (Muñoz Caro et al. 2006). The similar $3.4 \mu \mathrm{m}$ feature profile of Stardust particles is thus compatible with a form of $a-\mathrm{C}: \mathrm{H}$, but Raman spectroscopy is required to confirm such identification (see Sect. 3.3 for Raman characterization).

The bottom spectrum of Fig. 9 corresponds to a residue made from ice photoprocessing of $\mathrm{H}_{2} \mathrm{O}: \mathrm{CH}_{3} \mathrm{OH}: \mathrm{NH}_{3}=2: 1: 1$ ice at $12 \mathrm{~K}$, which was warmed up to room temperature, after correction using a local spline baseline. This band presents a characteristic broad double-peaked profile. Remarkably, that profile is preserved despite the different compositions of the starting ice mixture, within the parameter space of relevant interstellar/circumstellar ices (Muñoz Caro \& Dartois 2008). The spectra on top correspond to the same residue after annealing at different temperatures under high vacuum. It is observed that as the 


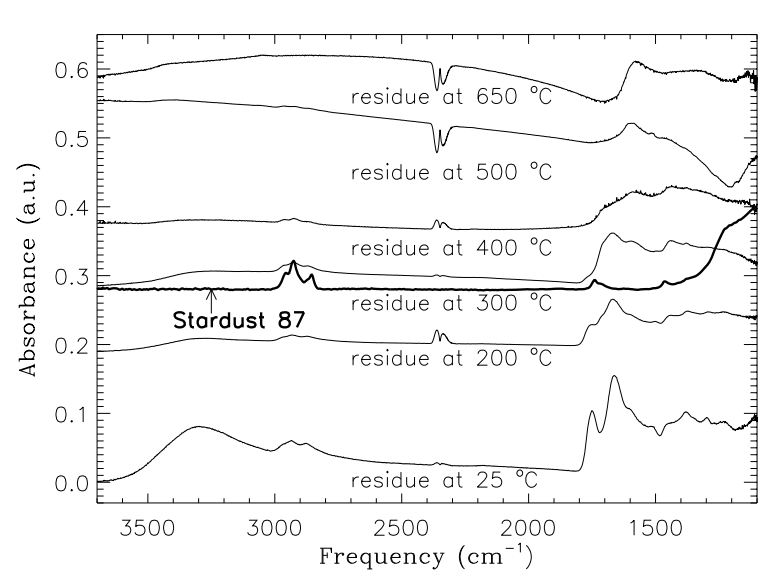

Fig. 10. Infrared spectra of an annealed organic residue made by UV irradiation of the $\mathrm{H}_{2} \mathrm{O}: \mathrm{CH}_{3} \mathrm{OH}: \mathrm{NH}_{3}=2: 1: 1$ ice mixture. The infrared spectrum of Stardust particle 87 (thick line) is shown for comparison.

annealing temperature increases, a band around $2965 \mathrm{~cm}^{-1}$ becomes visible, which corresponds to the aliphatic $\mathrm{CH}_{3}$ asymmetric stretching mode; this indicates a decrease in $N\left(\mathrm{CH}_{2}\right) / N\left(\mathrm{CH}_{3}\right)$ which is associated with the loss of $\mathrm{O}$ (and $\mathrm{N}$ ). The originally macromolecular organic residue became $a-\mathrm{C}: \mathrm{H}$. Finally, the spectrum at $650{ }^{\circ} \mathrm{C}$ shows no $3.4 \mu \mathrm{m}$ feature (aliphatic chains were lost); instead, a prominent band at $\sim 3050 \mathrm{~cm}^{-1}$ ( $3.3 \mu \mathrm{m}$ band assigned to the aromatic/olefinic $\mathrm{CH}$ stretch) is observed: the residue is progressively evolving toward graphitisation. Figure 9 shows that the $3.4 \mu \mathrm{m}$ feature of Stardust particles $\mathrm{C} 2054,0,35,16,0$ and 81 resemble that of the residue at $300-400{ }^{\circ} \mathrm{C}$.

Figure 10 compares the full mid-infrared spectrum of the annealed organic residue described above, which was produced by UV irradiation of the $\mathrm{H}_{2} \mathrm{O}: \mathrm{CH}_{3} \mathrm{OH}: \mathrm{NH}_{3}=2: 1: 1$ ice mixture, and that of Stardust particle 87 , which has the largest carbonyl $(\mathrm{C}=\mathrm{O})$ stretching band from our sampling. While the organic residue spectrum at room temperature is rich in bands attributed to functional groups, which diminish after annealing at higher temperatures, in agreement with the above observation based on the $3.4 \mu \mathrm{m}$ feature, the spectrum of particle 87 only displays a weak carbonyl band at $1740 \mathrm{~cm}^{-1}$. A band at $1465 \mathrm{~cm}^{-1}$, due to aliphatic $-\mathrm{CH}_{3}$ asym. bending and $-\mathrm{CH}_{2}$ - bending modes, is observed. Figure S5 of Sandford et al. (2006) shows the spectrum of a section from track 59, where the band due to a functional group, with no contribution from aerogel, quoting these authors, is a carbonyl feature. In our analysis, the spectrum of the aerogel piece outside tracks (Fig. 4) shows also a carbonyl band. Therefore, aerogel could also contribute to the weak carbonyl band observed in the infrared spectra of some of our Stardust grains.

\subsection{Characterization of the carbon structure of Stardust samples based on the Raman $D$ and $G$ bands}

We provide an interpretation of the Raman spectra published by Sandford et al. (2006), based on Ferrari \& Robertson (2000).

The first-order Raman D and G bands of two Stardust particles (labelled F1_1 and F1_2, adapted from Fig. 1 A of Sandford et al. 2006, that correspond to track 35, grain 30 and track 13, grain 1) are shown in Fig. 11 after subtraction of the continuum using a spline baseline. For comparison, the D and $\mathrm{G}$ bands of IDPs W7116B-N and L2021-K2 (labelled N and K2,

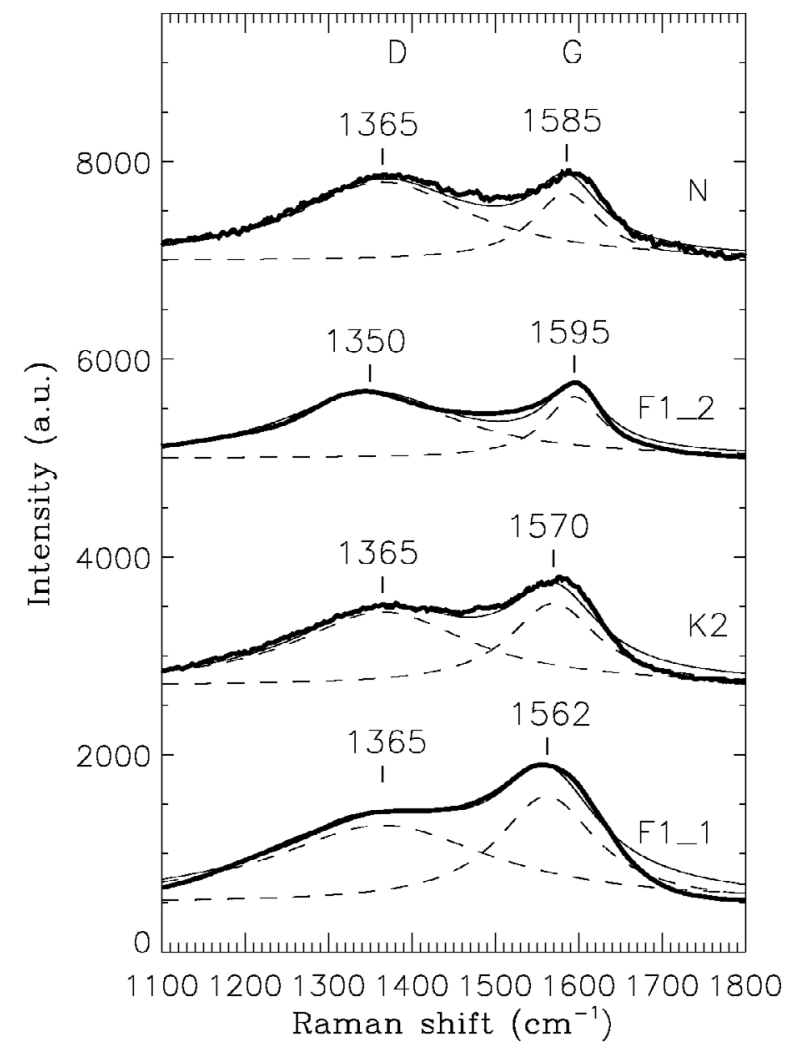

Fig. 11. Raman D and G bands of two Stardust particles (labelled F1_1 and F1_2, adapted from Fig. 1 A of Sandford et al. 2006) after soft smoothing and baseline correction. For comparison, the D and G bands of IDPs N and K2 (adapted from Fig. 3 of Muñoz Caro et al. 2006) are shown. Thin lines are the fits made by addition of two Lorentzians, plotted as dashed lines.

adapted from Fig. 3 of Muñoz Caro et al. 2006) are shown. The Lorentzian fits of the D and G bands, separately, and the sum of both Lorentzians, which provides a fit of the baseline-corrected first-order spectrum, are also provided. Spectra are well fitted apart from the excess of the fit on the blue wing of the $G$ band. Table 2 indicates the parameter values for the fits, namely the derived $\mathrm{D}$ and $\mathrm{G}$ line positions and FWHM, and the intensity ratio $I(\mathrm{D}) / I(\mathrm{G})$; it shows that all the parameter values of both Stardust particles fall within the parameter ranges of IDPs provided in the literature (Wopenka 1988; Ferini et al. 2004). The spectra of Stardust particles F1_1 and F1_2 resemble roughly those of IDPs K2 and N, respectively.

From Eq. (3) in Sect. 2.2 and the $I(\mathrm{D}) / I(\mathrm{G})$ values reported in Table 2, the aromatic domain sizes, $L_{\mathrm{a}}$, corresponding to Stardust particles F1_1 and F1_2 are 1.2 nm (5 rings in diameter, or a total number of $\sim 25$ rings assuming a 2-dimensional structure) and $1.4 \mathrm{~nm}$ (close to 6 rings in diameter, i.e. a total number of $\sim 30$ rings, containing $\sim 80 \mathrm{C}$ atoms, assuming a 2-dimensional structure).

In general, the aromatic domain size of amorphous carbon should be lower than $2 \mathrm{~nm}$ (Ferrari \& Robertson 2000). Using the $\mathrm{G}$ line position and FWHM values reported in that publication, and the $I(\mathrm{D}) / I(\mathrm{G})$ values of F1_1 and F1_2, it is found that the structure of the carbon bulk in both Stardust particles is that of $a-\mathrm{C}$ or $a-\mathrm{C}: \mathrm{H}$. The $3.4 \mu \mathrm{m}$ absorption feature of these particles in the infrared, is compatible with $a-\mathrm{C}: \mathrm{H}$ (Sect. 3.2). The Raman spectra of 12 Stardust particles are qualitatively similar to those of many IDPs and primitive meteorites (Sandford et al. 2006), that are known to contain $a-\mathrm{C}$ or $a-\mathrm{C}: \mathrm{H}$ (Muñoz Caro et al. 2006; 
Table 2. Lorentzian fit values for the $\mathrm{D}$ and $\mathrm{G}$ bands that correspond to line position, FWHM, and intensity ratio $I(\mathrm{D}) / I(\mathrm{G})$.

\begin{tabular}{cccccc}
\hline \hline IDP & $\begin{array}{c}\text { D line position } \\
\left(\mathrm{cm}^{-1}\right)\end{array}$ & $\begin{array}{c}\text { G line position } \\
\left(\mathrm{cm}^{-1}\right)\end{array}$ & $\begin{array}{c}\text { D line FWHM } \\
\left(\mathrm{cm}^{-1}\right)\end{array}$ & $\begin{array}{c}\text { G line FWHM } \\
\left(\mathrm{cm}^{-1}\right)\end{array}$ & $I(\mathrm{D}) / I(\mathrm{G})$ \\
\hline F1_1 (Stardust) & 1365 & 1562 & 322 & 146 & 0.73 \\
F1_2 (Stardust) & 1350 & 1595 & 248 & 86 & 1.06 \\
K2 (IDP) & 1365 & 1570 & 256 & 130 & 0.90 \\
N (IDP) & 1365 & 1585 & 256 & 100 & 1.17 \\
11 IDPs (average) ${ }^{1}$ & $1362(1345-1393)$ & $1584(1562-1594)$ & $296(141-476)$ & $104(72-159)$ & $1.02(0.66-1.39)$ \\
\hline
\end{tabular}

${ }^{1}$ Average values of Raman parameters corresponding to 11 IDPs (Wopenka 1988), reported by Ferini et al. (2004).

Muñoz Caro \& Martínez Frías 2007). This indicates that $a-\mathrm{C} / a-$ $\mathrm{C}: \mathrm{H}$ may be common in Stardust particles. The spectroscopic analysis of a large number of Stardust particles is required to estimate the amount of particles that contain $a-\mathrm{C}$ or $a-\mathrm{C}: \mathrm{H}$.

The presence of either $a-\mathrm{C}$ or $a-\mathrm{C}: \mathrm{H}$ in the particle may be indigenous to cometary grains or due to the degree of thermal annealing experienced during aerogel impact, following the sequence (in order of increasing temperature): macromolecular organic matter $\rightarrow a-\mathrm{C}: \mathrm{H} \rightarrow a-\mathrm{C} \rightarrow$ graphitisation.

Visible Raman spectroscopy (green laser, $\lambda=514.5 \mathrm{~nm}$ ) of organic residues produced by ice photoprocessing shows a high photoluminescence, which is presumably due to its organic nature with high $\mathrm{H} / \mathrm{C}$ and $\mathrm{O} / \mathrm{C}$ ratios, that masks the presence of the first-order D and G bands (Muñoz Caro et al. 2006, see bottom panel of Fig. 4). Thus, it is evident that this macromolecular organic material differs greatly from the amorphous carbon bulk of cometary Stardust samples that show clear D and G bands. The $D$ and $G$ bands only appear in the Raman spectrum of the organic residues after annealing at high temperatures: at $650{ }^{\circ} \mathrm{C}$ we found that the ratio $I(\mathrm{D}) / I(\mathrm{G})$ is 1.8 , which corresponds, using Eq. (3), to an aromatic domain size of $1.8 \mathrm{~nm}$. The Raman spectra of particles reported so far, show lower $I(\mathrm{D}) / I(\mathrm{G})$ values and correspond to less-ordered amorphous carbon. Therefore, if we assume that the carbon in cometary Stardust grains was organic prior to aerogel collection, these cometary grains were probably not exposed to temperatures above $\sim 650{ }^{\circ} \mathrm{C}$. The Raman D and $\mathrm{G}$ bands, which were attributed to cometary Stardust grains (Sandford et al. 2006), could correspond to the same carbon material that absorbs at $3.4 \mu \mathrm{m}$ in the infrared, although a band around $1600 \mathrm{~cm}^{-1}$ (or $6.2 \mu \mathrm{m}$, characteristic of the $\mathrm{C}=\mathrm{C}$ stretch in aromatics) is weak or absent in the infrared spectra of the particles, which suggests that the infrared carrier is mainly aliphatic. The analysis of the same particle by both infrared and Raman spectroscopy is required to determine whether both spectra are produced by the same carbonaceous carrier.

\section{Conclusions}

After substantial effort, we determined that the contribution of aerogel-carbon contamination to the infrared spectrum of our samples, was significant. We concluded that there is a mixture of cometary carbon and carbon from the aerogel, although it is difficult to determine quantitatively the contribution from individual sources. Hopefully, our approach, based on the determination of both the $N\left(\mathrm{CH}_{2}\right) / N\left(\mathrm{CH}_{3}\right)$ and $N[3.4] / N[10]$ ratios in particle and aerogel samples, can aid future works to constrain the contribution of aerogel carbon in their samples. For that purpose, we encourage the publication of the full mid-infrared spectrum of samples, so that the $N[3.4] / N[10]$, i.e. the aliphatic C-to-aerogel $\mathrm{Si}$ ratio, can be calculated. Since cometary carbon should be concentrated mainly in the track, and partly in the surrounding halo, this ratio should decrease as a function of distance away from the track; caution is needed, however, in the case of cometary samples with a considerable intrinsic silicate-to-aliphatic carbon ratio, since, in that case, the $10 \mu \mathrm{m}$ feature has a contribution from the $\mathrm{Si}-\mathrm{O}$ bonds in both aerogel and indigenous cometary silicates.

It was found that, based on Raman spectroscopy, the carbon bulk of the Stardust particles reported so far by Sandford et al. (2006) corresponds to $a-\mathrm{C}$ or $a-\mathrm{C}: \mathrm{H}$. This material is generally described as a poorly-graphitized carbon with an aromatic domain size smaller than $2 \mathrm{~nm}$. For the Stardust particles reported by Sandford et al. (2006), we estimated aromatic domain sizes of 1.2 and $1.4 \mathrm{~nm}$, which correspond to $\simeq 5-6$ rings in diameter. Such aromatic units are probably linked either by aliphatic chains for Stardust particles containing $a-\mathrm{C}: \mathrm{H}$, which have $N\left(\mathrm{CH}_{2}\right) / N\left(\mathrm{CH}_{3}\right)$ ratios around 4 , assuming that those particles are also responsible for the $3.4 \mu \mathrm{m}$ feature observed in the infrared, or a carbon $\mathrm{sp}^{2}$ and $\mathrm{sp}^{3}$-skeleton for Stardust particles containing $a$-C. A schematic of the expected substructure unit of the $a-\mathrm{C}: \mathrm{H}$ material, which is thought to be present in Stardust particles and a large population of IDPs, is depicted in the top panel of Fig. 12.

It was proposed that the high $\mathrm{O}$ and $\mathrm{N}$ contents, lower aromatic contents with respect to carbonaceous matter in diffuse interstellar grains, and elevated $-\mathrm{CH}_{2}-/-\mathrm{CH}_{3}$ ratios of Stardust organics are qualitatively consistent with radiation processing of astrophysical ices in the dense cloud and/or the protosolar nebula (Sandford et al. 2006).

It is important to note that the amorphous carbon of Stardust particles can incorporate functional groups such as $\mathrm{C}=\mathrm{O}$, which are observed in the infrared. In agreement with Sandford et al. (2006), it was found that the $-\mathrm{CH}_{2}-/-\mathrm{CH}_{3}$ ratio of Stardust samples is similar to IDPs and stands well above that of carbon grains observed toward the diffuse interstellar medium. The hydrogenated part of the material that absorbs at $3.4 \mu \mathrm{m}$, in diffuse interstellar carbon grains, was characterized as $a-\mathrm{C}: \mathrm{H}$ with small aromatic units of 1-2 rings, which was linked by aliphatic chains responsible for a $N\left(\mathrm{CH}_{2}\right) / N\left(\mathrm{CH}_{3}\right)$ ratio around 2 (compared to $\sim 4$ in cometary Stardust grains), being $\mathrm{O}$ and $\mathrm{N}$ poor (Dartois et al. 2005); see bottom panel of Fig. 12. However, Sandford et al. (2006) stated that Stardust organics have lower aromatic contents than diffuse interstellar carbon grains. The aromatic content with respect to the total mass of the particle, is difficult to estimate given the unavailability of diffuse interstellar carbon grains for in situ analysis in the laboratory. We compared instead the structure of the hydrogenated carbon material (Fig. 12). We observed that the dominant carbon in Stardust samples was more ordered than diffuse interstellar carbon, which are aromatic units that contain more than 20 rings compared to 1-2 rings in diffuse interstellar grains. Compared to aliphatic $\mathrm{CH}$ stretching absorptions, strong aromatic $\mathrm{CH}$ bands are observed neither in the interstellar medium lines-of-sight, nor in spectra of Stardust grains, but a band attributed to the backbone of $\mathrm{C}=\mathrm{C}$ is observed 

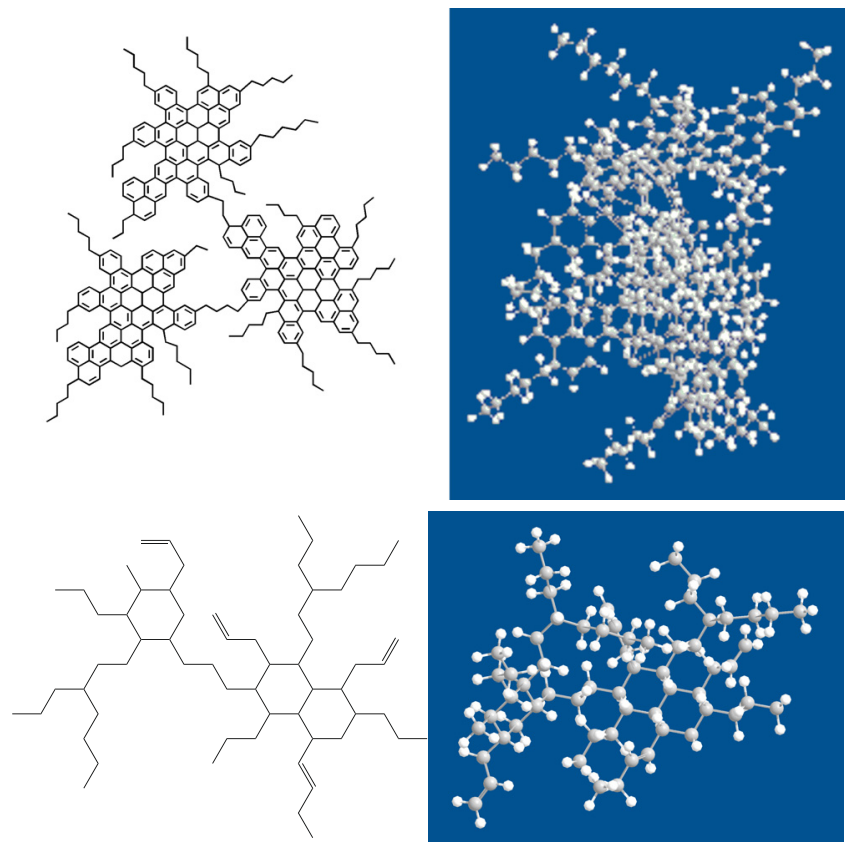

Fig. 12. Top left: a schematic of the expected substructure unit for the $a$-C:H polymer observed in Stardust grains and many IDPs showing 3 aromatic units depicted in 2 dimensions. Top right: the real structure is not planar because it folds gradually as the number of aromatic units increases. This $a-\mathrm{C}: \mathrm{H}$ polymer is build up of numerous aromatic units linked by aliphatic chains, which lead to the intricate structure characteristic of $a-\mathrm{C}: \mathrm{H}$. Functional groups $(\mathrm{OH}, \mathrm{C}=\mathrm{O}$, etc.; not shown in this figure) can be inserted in the network. Figure adapted from Muñoz Caro \& Dartois (2007). Bottom left: a schematic of the expected substructure unit for the photoproduced $a-\mathrm{C}: \mathrm{H}$ (analog of the carbonaceous material in diffuse interstellar grains), which was adapted from Dartois et al. (2005). Bottom right: the photoproduced $a-\mathrm{C}: \mathrm{H}$ polymer is also a complex 3-dimensional structure.

in some extragalactic interstellar medium lines-of-sight (Dartois et al. 2007).

We showed that the cometary Stardust grains reported so far, are spectroscopically distinct from organic residues made from ice photoprocessing: these residues were not subsequently annealed above room temperature at $300-400{ }^{\circ} \mathrm{C}$, which would have caused them to lose part of the $\mathrm{O}$ and $\mathrm{N}$ content and suffer a structural reorganisation. We conclude that the dominant carbon detected in Stardust grains, which show D and G Raman bands (Sandford et al. 2006), is $a-\mathrm{C}$ or $a-\mathrm{C}: \mathrm{H}$, while the residues made from ice photoprocessing are a macromolecular organic material. Therefore, the carbon fraction of cometary grains which show D and G Raman bands, or the observed $3.4 \mu \mathrm{m}$ infrared feature compatible with $a-\mathrm{C}: \mathrm{H}$, is not a direct product of ice photoprocessing.

Stardust proved that cometary particles can be collected in large numbers. We propose that a future mission should employ a collecting material as free from carbon contamination as possible, and collect cometary particles that impact at lower velocities to preserve more effectively the original composition of the grains.

Acknowledgements. We are grateful to M. E. Zolensky and his colleagues at the NASA JSC Curatorial Facility for kindly providing the IDPs used in this work. We thank Oscar and Sofía Aparicio for discussions. G.M.M.C. was supported by a Ramón y Cajal research contract from the MCYT in Spain.

\section{References}

Allamandola, L. J., Sandford, S. A., \& Valero, G. 1988, Icarus, 76, 225 Bernstein, M. P., Dworkin, J. P., Sandford, S. A., Cooper, G. W., \& Allamandola, L. J. 2002, Nature, 416, 401

Bregman, J. D., Campins, H., Witteborn, F. C., et al. 1987, A\&A, 187, 616

Brownlee, D., Tsou, P., Aléon, J., et al. 2006, Science, 314, 1711

Dartois, E., Marco, O., Muñoz Caro, G. M., et al. 2004, A\&A, 423, 549

Dartois, E., Muñoz Caro, G. M., Deboffle, D., Montagnac, G., \& d'Hendecourt, L. 2005, A\&A, 432, 895

Dartois, E., Geballe, T. R., Pino, T., et al. 2007, A\&A, 463, 635

Domínguez, G., Westphal, A. J., Jones, S. M., \& Phillips, M. L. F. 2004, Icarus, 172,613

Domínguez, G., Trigo-Rodríguez, J. M., Burchell, M. J., et al. 2007, Meteoritics \& Planet. Sci. Suppl., 42, 5235, A37

Ferini, G., Baratta, G. A., \& Palumbo, M. E. 2004, A\&A, 414, 757

Ferrari, A. C., \& Robertson, J. 2000, Phys. Rev. B, 61, 20, 14095

Greenberg, J. M. 1998, A\&A, 330, 375

Hanner, M. S. 1999, Space Sci. Rev., 90, 99

Hörz, F., Cintala, M. J., Zolensky, M. E., et al. 1998, NASA/TM-98-201792, 58

Hörz, F., Bastien, R., Borg, J., et al. 2006, Science, 314, 1716

Hurler, W., Pietralla, M., \& Hammerschmidt, A. 1995, Diamond and related materials, 4, 954

Keller, L., Bajt, S., Baratta, G. A., et al. 2006, Science, 314, 1728

Matrajt, G., Muñoz Caro, G. M., Dartois, E., et al. 2005, A\&A, 433, 979

Matrajt, G., Ito, M., Wirick, S., et al. 2007, MAPS, in press

McKeegan, K. D., Aleon, J., Bradley, J., et al. 2006, Science, 314, 1724

Meierhenrich, U. J., Muñoz Caro, G. M., Schutte, W. A., et al. 2004, Chem. Eur. J., 11, 4895

Muñoz Caro, G. M., \& Dartois, E. 2007, Adv. in Geosciences, Planetary Sciences (2006), ed. A. Bhardwaj, et al., 7, 155

Muñoz Caro, G. M., \& Dartois, E. 2008, in prep.

Muñoz Caro, G. M., \& Martínez-Frías 2007, Carbonaceous Dust in Planetary Systems: Origin and Astrobiological Significance, in Proceedings of Dust in Planetary Systems 2005, Kauai, Hawaii, USA, ESA SP-643, Jan. 2007, 133 Muñoz Caro, G. M., \& Schutte, W. A. 2003, A\&A, 412, 121

Muñoz Caro, G. M., Meierhenrich, U. J., Schutte, W. A., et al. 2002, Nature, 416, 403

Muñoz Caro, G. M., Matrajt, G., Dartois, E., et al. 2006, A\&A, 459, 147

Nakamura-Messenger, K., Messenger, S., Keller, L. P., Clemett, S. J., \& Zolensky, M. E. 2006, Science, 314, 1439

Okudaira, K., Noguchi, T., Nakamura, T., et al. 2004, Adv. Space Res., 34, 2299 Oró, J. 1961, Nature, 190, 389

Raynal, P. I., Quirico, E., Borg, J., et al. 2000, Planet. Space Sci., 48, 1329

Sandford, S. A., \& Brownlee, D. E. 2007, Science, 317, 1680d

Sandford, S. A., Aléon, J., Alexander, C. M. O’D., et al. 2006, Science, 314, 1720

Spencer, M. K., \& Zare, R. N. 2007, Science, 317, 1680c

Titantah, J. T., Lamoen, D., Neyts, E., \& Bogaerts, A. 2006, J. Phys. Cond. Matt., 18,10803

Tsou, P., Brownlee, D. E., Sandford, S. A., Hörz, F., \& Zolensky, M. E. 2003, J. Geophys. Res., 108, 8113

Tuinstra, F., \& Koenig, J. L. 1970, J. Chem. Phys., 53, 23

Wolf, A., \& Brendel, R. 2006, Thin Solid Films, 513, 385

Wopenka, B. 1988, Earth. Planet. Sci. Lett., 88, 221 